\title{
THE FASCIAL SPACES OF THE NECK IN ACUTE INFECTION*
}

\section{FREDerick A. Coller, M.D.}

Professor of Surgery, University of Michigan School of Medicine
AND

Resident in Surgery, University of Michigan School of Medicine

\section{ANN ARBOR, MICHIGAN}

$A^{\mathrm{LT}}$ THOUGH the importance of fascial planes in acute infections of the extremities is well understood by most physicians, the relation of the spread of infection in the cervical region to the fascial architecture is much less appreciated. The course of infections of the neck is determined by definite fascial planes that may act as barriers to localize infection or may direct the spread of an acute suppurative process to involve fascial compartments in the mediastinum. Hare states that of seventy-eight cases of pyogenic mediastinal suppurations, seventeen, or 22 per cent, were of cervical origin. Pearse collected I Io cases of suppurative mediastinitis and found perforation of the cervical esophagus accountable for 58. I per cent, suppurative cervical adenitis I .8 per cent, retropharyngeal abscess Io per cent, peritonsillar abscess 7.2 per cent, tracheotomy 5.5 per cent, spondylitis 2.8 per cent, thyroidectomy 2.8 per cent, and Ludwig's angina 1.8 per cent.

If the superficial types of infections such as furuncles are omitted, infections of the neck may be divided into four general groups. In this paper, only Group One will be discussed.

I. Acute suppurations secondary to infections elsewhere. Of this group, Boemer reviewed seventy-five cases and found thirty-eight from retropharyngeal abscess, eleven from deep cervical glands, nine from lower third molar, seven from superfrcial cervical glands, three from tonsils, two from mastoid, and five from miscellaneous sources including carcinoma and fractures.

2. Specific infections such as tuberculosis, syphilis, actinomycosis, blastomycosis, and tularemia.
3. Infected cysts and tumors, including cystic hygroma, Iymphangioma, and degenerating malignant nodes.

\section{Thyroiditis.}

Fascial planes classified by their relation to the spread of infection may be divided into two groups: those associated with muscles, and those surrounding viscera and vessels. The muscular fascial planes are always ultimately inserted into bone, thereby sharply limiting infections in these spaces. The viscerovascular fascial spaces extend along vessels and viscera in continuity with these structures, thereby allowing infection in them to pass readily from one region to another. In the face, three muscular fascial spaces have been described: the space of the body of the mandible, the masticator space, and the parotid space, and one viscerovascular space, the pterygopharyngomaxillary or the lateral pharyngeal space.

In the neck there are also definite fascial planes of both types. After the skin, subcutaneous tissue, and platysma muscles have been reflected, the first muscular fascial plane of the neck encloses the sternocleidomastoid and trapezius muscles. This fascia is continuous with the fascial boundaries of the masticator space, and blends with the periosteum of the clavicle and sternum. Deep to this plane is found the second muscular fascial plane in which lie the sternohyoid and omohyoid muscles. Above it joins the first layer of fascia at the hyoid base to form the fused fascia, while below it splits at the sternum into two layers to form the suprasternal space. Laterally it blends with the periosteum of the posterior border of the clavicle and with the upper border of the scapula where the omohyoid is inserted. The plane then

\footnotetext{
* From the Department of Surgery, University of Michigan.
} 
continues around the neck between the trapezius and the deep muscles of the neck to insert into the ligamentum nuchae. The posteriorly surrounds the aorta. Laterally it passes with the intercostal arteries to merge with the parietal pleura. This large

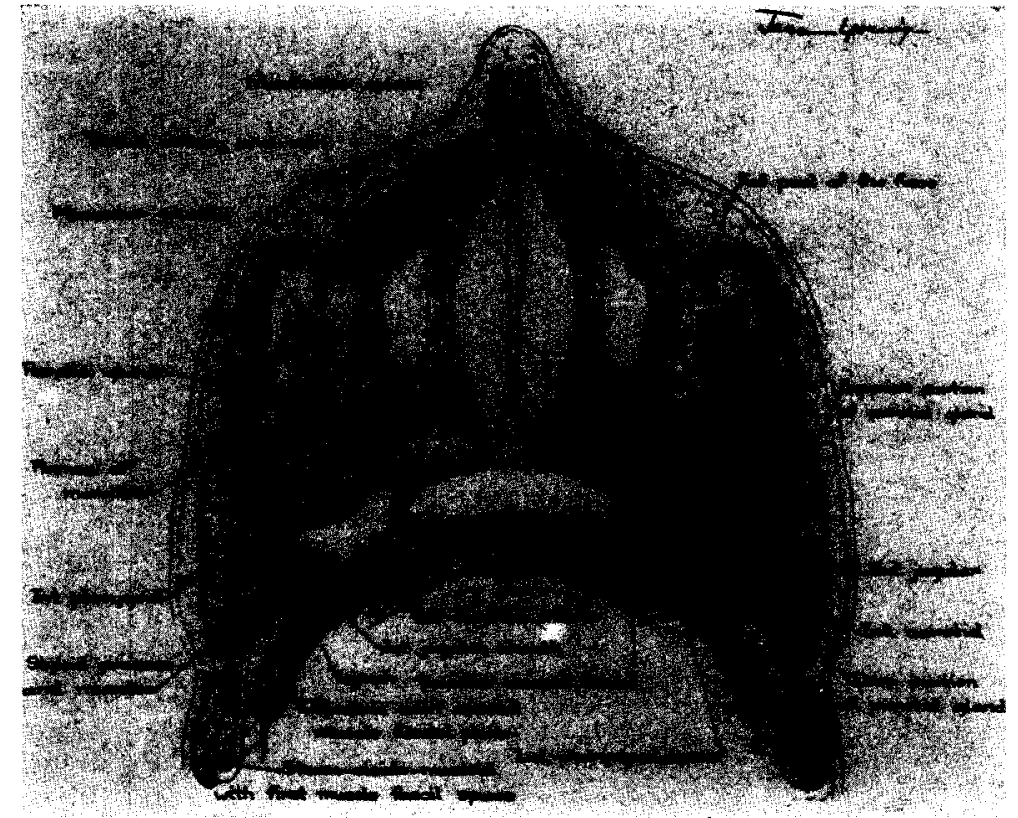

FIG. I. Horizontal section demonstrating the different fascial planes and potential anatomic spaces. (From Coller and Yglesias, in Surg., Gynec. of Obst., 60: 277, 1935.)

suprasternal space is not important as far as infections of the neck are concerned. It may be infected through stab wounds, osteomyelitis of the hyoid bone and from the lymphatics. The third muscular fascial layer is composed of the fascia enclosing the sternothyroid and thyrohyoid muscles; it continues above to the hyoid bone, laterally to fuse with the fascial sheath of the internal jugular vein, and inferiorly it is attached to the posterior border of the manubrium and first rib. This space is seldom infected and its only practical application is that the surgeon following this space laterally will meet the internal jugular vein.

The viscerovascular fascia, as its name implies, envelops the viscera of the neck and mediastinum, carotid sheath and aorta, forming a cylindric covering of the pharynx, Iarynx, aorta, and its branches which ascend into the neck. Within the thoracic cavity this fascial layer blends anteriorly with the pericardial sac and space is of the greatest importance to the surgeon since it connects the mouth, throat, and pharynx and is the route along which infections commonly pass. For a better understanding of this region, it may be divided into several parts.

The fascia lying posterior to the pharynx has been called the buccopharyngeal fascia; the layer lying anterior to the trachea, the pretracheal fascia; and that portion surrounding the aorta and its branches, the vascular sheath. The lateral pharyngeal space, which may be infected from any of the three important fascial spaces of the face and from the pharynx and tonsil, can act as portal of entry to the large viscerovascular space.

The submaxillary gland is enclosed by that portion of the viscerovascular fascia that surrounds the pharynx and covers the floor of the mouth. This gland with its coverings is separated from the parotid space and subcutaneous tissue by the fused fascia of the first and second muscu- 
lar fascial planes of the neck. Posteriorly the fascia covering the gland is connected to the external carotid sheath and forms the buccopharyngeal fascia of the pharynx and the retroesophageal fascia of the esophagus. Infections arising from the

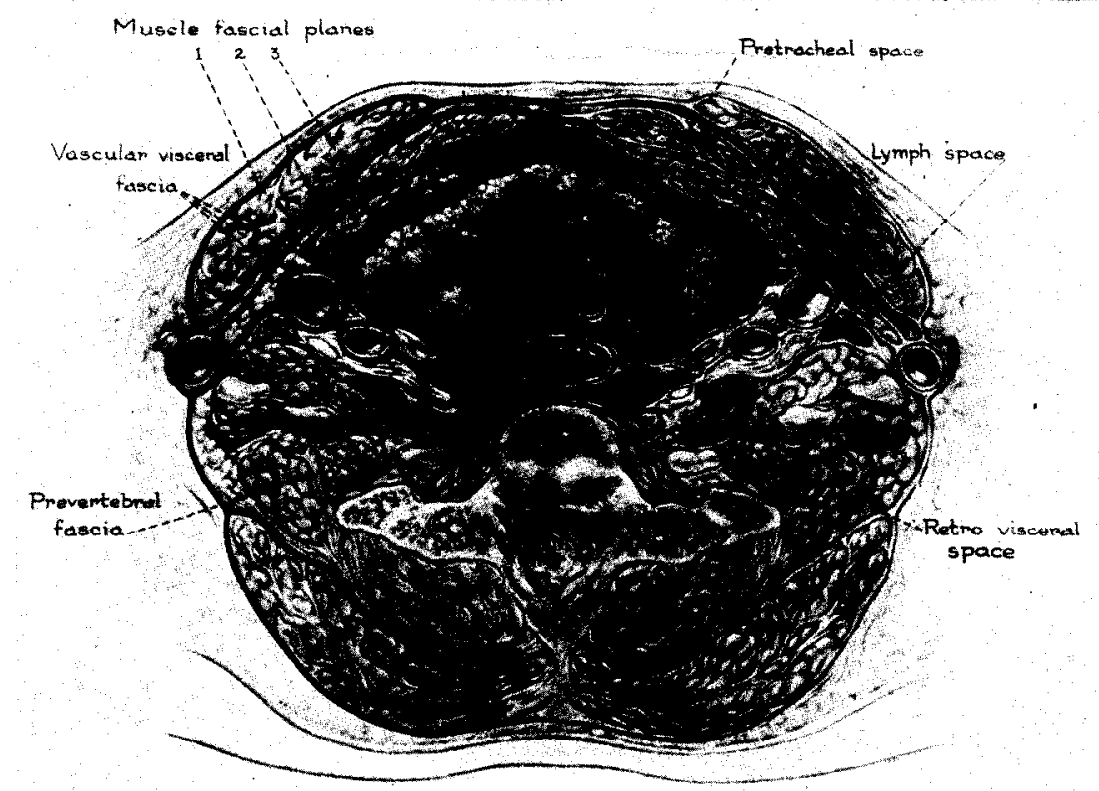

FIG. 2. Horizontal scction at the neck showing disscetion of the fascial plancs and spaces. (From Coller and Yglesias, in Surgery, I: 323, 1937.)

the floor of the lateral pharyngeal spacc. Infections of the submaxillary gland may perforate and infect the viscerovascular space. Diagnosis is not difficult. The infection manifests itself as a hard, tender swelling over the submaxillary triangle. The mouth is opened with diffrculty and movement of the tongue is painful. Although some of these deep infections subside with conservative treatment, if the patient continues to run a septic course after twentyfour to forty-eight hours, operation is indicated. Removal of the gland has proved the best method of attack.

The pretracheal fascia is that fascia which encloses the larynx, trachea, and thyroid gland. It descends into the mediastinum surrounding the tracheobronchial Iymphatic system, the arch of the aorta, and the right branch of the pulmonary artery to blend finally with the pericardial sac. Laterally in the neck, the fascia merges with the sheath of the common carotid artery. Posteriorly it continues to form lateral waIl of the pharynx, such as peritonsillar abscess or infections of the lateral pharyngeal space, may extend down to infect the entire pretracheal space. Perforations of the anterior wall of the esophagus may also reach this space. That portion of the viscerovascular fascia surrounding the carotid artery and internal jugular vein, forms the true sheath for these vessels and may be infected from the lateral pharyngeal space, but if this occurs the infection usually remains limited to the sheath.

The fascia that covers the posterior wall of the pharynx and esophagus is the retrovisceral portion of the viscerovascular system. Between this and the prevertebral fascia is a large and important space bounded Iaterally in the neck by the fusing of these fasciae at the point where they surround the cervical sympathetic chain. Above it extends to the basilar process of the skull and is continuous below to the diaphragm. In the thorax the Iateral 
boundaries are formed by the expansion of the sheath covering the descending aorta as it passes posterior to the parietal pleura fection of the retrovisceral space passing downward to expand laterally between the pleurae and the lateral expansion of the

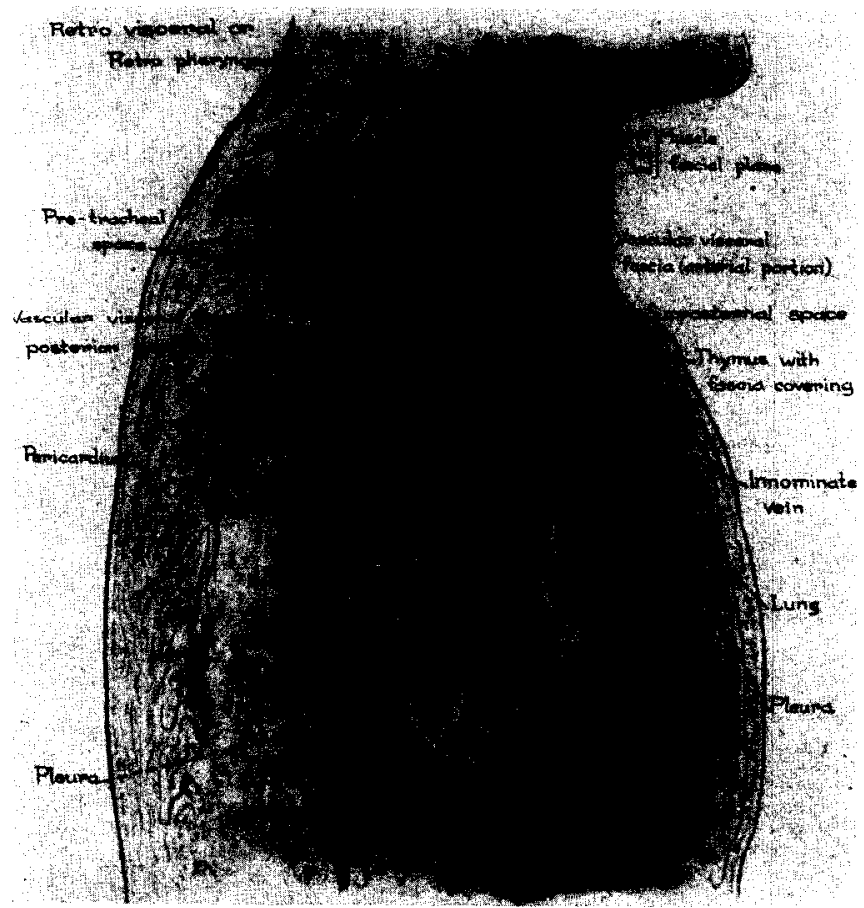

FIg. 3. Sagittal section through neck and thorax showing fascial planes and spaces. (From Coller and Yglesias, in Surgery, $1: 323$, 1937.)

to join the posterior wall of the thorax. Below the level of the bifurcation of the trachea, the space becomes very small and may be occluded by the close approximation of the two pleurae. The clinical importance of this pathway is obvious. Its intimate relation to the vertebrae, esophagus, and lymph nodes exposes it to infection from all sides. Pyogenic and tuberculous infection arising from any bone adjacent to this area, including the basilar process of the skull, the petrous portion of the temporal bone, the cervical and dorsal vertebrae, and the posterior ends of the ribs, may spread to this space.

Infections in any of the cervical and upper dorsal vertebrae will first produce a prevertebral abscess that is limited by the prevertebral fascia, muscles, and ligaments, and will remain localized here for varying lengths of time. Eventually, however, perforation will occur with secondary in- aortic sheath as it attaches to the thoracic wall. If the infection originates in the posterior end of the ribs, the spread of the infection will be in a reverse direction from the ribs along the space between the pleura and the lateral expansion of the sheath of the descending aorta to the retrovisceral space. For these reasons, infections in the retrovisceral space above the fourth dorsal vertebra, may best be drained by an incision in its upper portion in the neck, and infections below this point must be drained by dorsal mediastinotomy effected by posterior rib resection.

Clinically, this abscess will give rise to a variety of well known signs and symptoms, depending upon its point of origin. Dysphagia, dyspnea, dysphonia, and a bulge in the posterior pharyngeal wall are generally seen. Infants and children will have as their chief symptom dyspnea, since the posterior wall of the trachea is less 
frxed and the trachea is more easily compressed than in adults. In adults dysphagia will often be the frrst symptom. $X$-ray examination will show an increase in width of the retropharyngeal space in both the frontal and lateral projections.

Drainage of infection in the retrovisceral space may be carried out through an incision made along the anterior border of the sternocleidomastoid muscle at any desired level between the hyoid bone and the sternum, depending upon the level of the infection. Cervical mediastinotomy is carried out by division of the skin, subcutaneous tissues, and platysma muscle; the first muscular fascial plane is divided along the anterior border of the sternocleidomastoid muscle; the second muscular fascial plane is divided along the lateral border of the omohyoid muscle; and the third muscular fascial plane is incised and separated from the sheath of the internal jugular vein. The lateral or lower border of the thyroid gland is lifted, and at the lower border of the thyroid is encountered the fascia passing from the thyroid to the venous portion of the viscerovascular fascia and to the thymus gland. After incising this fascia, the upper portion of the pleural-pericardial space has been opened and drainage is accomplished. This approach is best carried out on the right side, as the pleura and esophagus meet at a lower level than on the left, consequently there is less chance of inadvertently opening the pleura during the dissection. Postural drainage and intermittent suction at frequent intervals are helpful after the drainage tube is placed. In early infection of the space, incision through the posterior pharyngeal wall at point of maximum swelling will usually be adequate, but if the infection has passed down, or originated in the lower portion of the space below the fourth cervical vertebra, drainage will necessarily be carried out by external thoracotomy.

Infection of the cervical lymph glands is often encountered by the surgeon. The superficial group of glands lies under the platysma muscle along the external jugular vein. When infected, they usually form localized subcutaneous abscesses and do not communicate with other fascial spaces of the neck. The deep group of nodes, however, lies along the great vessels of the neck in a space bounded anteriorly by the sheath of the internal jugular vein and common carotid artery, posteriorly by the fascia covering the anterior scalene muscle. The space extends upward to the mastoid and continues down to the supraclavicular fossa. Here the nodes end in the angle between the subclavian and internal juguIar veins. Infection extending down this chain of nodes may pass directly to the axilla.

\section{SUMMARY}

A description of the fascial spaces of the neck with practical application to the routes of infection has been given. The importance of a knowledge of the fasciae and fascial spaces in anticipating the spread of infection is obvious.

\section{REFERENCES}

I. Barnhill, J. F. Deep abscess of the neck. Am. J. Surg., 42: 207-220, 1938.

2. Boemer, L. C. The great vessels in deep infection of the neck. Arcb. Otolaryngl., 25:465-472, 1937.

3. Coller, F. A., and Yglesias, L. Infections of the lip and face. Surg., Gynec. of Obst., 60: 277-290, 1935.

4. Coller, F. A., and Yglesias, L. The relation of the spread of infection to fascial planes in the neck and thorax. Surgery, $1: 323-338,1937$.

5. Colp, R. Treatment of deep infections of submaxillary triangle. Surg., Gynec. of Obst., 68: IIO2-1 103, 1939.

6. Furstenberg, A. C. Acute suppurations of throat, mouth and cervical region. Tr. Oto-Opbtbal. Soc., 2I: $14-25,1936$.

7. Furstenderg, A. C. Acute mediastinal suppuration. Tr. Am. Laryng., Rbin. of Otol. Soc., 35: 210 , 1929.

8. Furstenberg, A. C., and Yglesias, L. Mediastinitis. Arcb. Otolosyngol., 25: 539-554, 1937.

9. Grodinsky, Manuel. The fasciae and fascial spaces of the head, neck and adjacent regions. Am. J. Anat., 63: 367-408, 1938.

Io. Hare, H. A. Pathology, Clinical History and Diagnosis of Infections of the Mediastinum. PhiladeIphia, 1887. Blakiston.

II. Haven, F. Z. Infections of the neck. Arch. Otolaryng., 21 : 536-54I, 1935.

12. Pearse, H. E. Mediastinitis following cervical suppuration. Ann. Surg., 108: 586-612, 1938. 